poor mental health when data can be linked to them, even if their personal details are only used to help them access care. This may be particularly relevant because individuals who have a mental health problem are more likely to experience barriers to care and hold stigmatising beliefs. If that is the case, then mental health screening programmes where personal details are required may not be effective in detecting those most in need of care. We aimed to compare mental health symptom reporting when using an anonymous versus identifiable questionnaire among UK military personnel on deployment in Iraq (early 2009).

Methods This was a survey among UK military personnel using two questionnaires, one anonymous $(n=315)$ and one identifiable $(n=296)$. Questionnaires were distributed by alternative allocation. The questionnaire included the 12-item General Health Questionnaire (measuring symptoms of common mental disorder, CMD), the Post-Traumatic Stress Disorder (PTSD) Checklist Civilian Version (measuring probable PTSD) and 11 stigma statements relating to barriers of care and perceived social stigma.

Results Of 612 personnel approached to take part, 99.8\% completed the survey. The overall prevalence of probable PTSD was $3.3 \%$ and $20.5 \%$ for symptoms of CMD. No significant difference in the reporting of symptoms of CMD was found (18.1\% identifiable vs. $22.9 \%$ anonymous, $\mathrm{P}=0.150)$. Personnel were more likely to report borderline and probable PTSD when completing questionnaires anonymously (borderline PTSD: $2.4 \%$ identifiable vs. $5.8 \%$ anonymous; probable PTSD: $1.7 \%$ identifiable vs. $4.8 \%$ anonymous, $\mathrm{P}=0.022$ ). Of the 11 barriers to care and perceived social stigma statements considered, those completing the anonymous questionnaire were more likely to endorse: "leaders discourage the use of mental health services" ( $9.3 \%$ vs. $4.6 \%, \mathrm{P}=0.029)$, "it would be too embarrassing" (41.6\% vs. $32.5 \%, \mathrm{P}=0.023)$ and "I would be seen as weak" (46.6\% vs. $34.2 \%, \mathrm{P}=0.003)$.

Conclusion We found a significant effect on the reporting of PTSD and certain stigmatising beliefs (but not $\mathrm{CMD}$ ) when using an anonymous compared to identifiable questionnaire. Our findings have implications for the current post-deployment screening policy used in the US militaryin which identifiable data are collected. These results suggest that researchers need to weigh up the balance between full anonymisation against the use of non-anonymised but confidential survey methods, which permit future follow up.

\section{OP92 THE IMPACT OF MILITARY DEPLOYMENT, COMBAT EXPERIENCES AND POST-DEPLOYMENT MENTAL HEALTH PROBLEMS ON VIOLENT BEHAVIOUR AMONG UK MILITARY PERSONNEL}

doi:10.1136/jech-2012-201753.092

1.2D MacManus, ${ }^{1} \mathrm{~K}$ Dean, ${ }^{2} \mathrm{M}$ Jones, ${ }^{2} \mathrm{R}$ Rona, ${ }^{2} \mathrm{~L}$ Hull, ${ }^{2} \mathrm{~N}$ Greenberg, ${ }^{\top} \mathrm{T}$ Fahy, ${ }^{2} \mathrm{~S}$ Wessely, ${ }^{2} \mathrm{~N}$ Fear. ${ }^{1}$ Forensic and Neurodevelopmental Sciences, King's College London, London, UK; '2King's Centre for Military Health Research, King's College London, London, UK

Background There is considerable media, political and public interest on both sides of the Atlantic in an alleged rise in violence among military personnel returned from conflicts in Iraq and Afghanistan. This study explores violence among a large sample of UK military personnel, a proportion of whom had been deployed to Iraq/Afghanistan. The aims were to estimate the prevalence of selfreported violence, examine the impact of deployment and combat experiences on subsequent violence and the association with postdeployment mental health problems and alcohol misuse.

Methods This study formed part of a questionnaire-based cohort study established to explore the impact of deployment on mental health among UK military personnel. The sample was randomly selected from all military personnel serving in the UK Armed Forces between 2003 and 2007. A total of 9986 participants from all Services were included, some had been deployed and some had been trained but not deployed. Special Forces were excluded. Data, collected by questionnaire, included information on deployment experiences, socio-demographic and military characteristics, preenlistment antisocial behaviour, and post-deployment health outcomes. The main violence outcomes were self -report measures of interpersonal violence.

Results $6.2 \%$ of the sample reported interpersonal violence in the last month. Deployment showed a stronger association with subsequent violence among reservists $(\mathrm{OR}=2.98$ (1.50-5.93), $\mathrm{P}=0.002)$ than among regulars $(\mathrm{OR}=1.26(0.96-1.67), \mathrm{P}=0.098)$ when compared to their non-deployed counterparts. Performing a combat role whilst deployed was associated with a significantly increased likelihood of violence among regulars $(\mathrm{OR}=1.90(1.38-2.75), \mathrm{P}<0.001)$ and the risk of violence increased with the number of traumatic events experienced $(\mathrm{P}<0.001)$ ). Violence on homecoming was also associated with mental health problems such as PTSD (OR $=4.8$ (3.2-7.2) $\mathrm{P}<0.001)$ and alcohol misuse $(\mathrm{OR}=3.1(2.5-3.9) \mathrm{P}<0.001)$.

Conclusion Military deployment, in particular combat exposure, increased the risk of subsequent violence among military personnel and the risk of violence increased with increasing number of traumatic events experienced on deployment. Valuable information on risk factors for violence among military personnel is provided, especially regarding increased risk among deployed reservists and among personnel who report post-deployment mental health problems and alcohol misuse.

\section{HSR: Evaluation of Health Care Interventions}

\section{OP93 ORLISTAT AND THE RISK OF ACUTE LIVER INJURY: A SELF- CONTROLLED CASE-SERIES STUDY IN UNITED KINGDOM GENERAL PRACTICE RESEARCH DATABASE}

doi:10.1136/jech-2012-201753.093

J Langham, L Smeeth, R Brauer, K Bhaskaran, I Douglas. Non Communicable Disease Epidemiology, LSHTM, London, UK

Background In 2009, based on spontaneous reports of serious liver injury the US Food and Drug Administration announced Orlistat may be linked to an increased risk of hepatic events. However, no causal association has been established. The aim of this study was to investigate the association between Orlistat and the incidence of acute liver injury.

Methods This was a self-controlled case-series design using the United Kingdom General Practice Research Database (GPRD) and linked Hospital Episode statistics (HES). People were eligible if they had an incident occurrence of idiopathic acute liver injury with a diagnoses recorded (in GPRD or HES) and were exposed to Orlistat at any time in the observation period. If there was evidence of a known cause for liver disease, such as alcoholism, patients were excluded. Observation time for each patient was divided into strata determined by Orlistat exposure status (30 day strata) and current age. Within-person rate ratios (with $95 \%$ confidence intervals) for liver injury were estimated using conditional Poisson regression (Stata 12), comparing exposed with unexposed periods.

Results In the GPRD, between 1999 and 2010, 94,695 people had received at least one prescription for Orlistat, of whom 1,741 had an eligible diagnosis recorded. Of these, 408 people fulfilled eligibility criteria for a definite event (including abnormal liver function test results and a referral). We found a higher incidence of events in the first 30 days of exposure, (compared to unexposed) RR 2.27 (95\% CI 1.12 to 4.59$)$ and in the 90 day pre-exposure period RR $1.96(95 \% \mathrm{CI}$ 1.35 to 2.85). There was no difference in the incidence of events between 90 days prior and 0-90 days post prescribing, RR 0.78 (95\% CI 0.42 to 1.42 ).

Conclusion This is the first study we are aware of to explore the risk of incident liver injury associated with Orlistat. We found an 